\title{
Image Smoothing By Low Gradient Minimization
}

\author{
V.Nancharaiah ${ }^{1}$, Sk.Azeez ${ }^{2}$, N.Raja Sekhar ${ }^{3}$ \\ ${ }^{1}$ Associate Professor, ECE Department, Lendi Institute Of Engineering and Technology, Jonnada, Vzm \\ ${ }^{2,3}$ Assistant Professor, ECE Department, Lendi Institute Of Engineering And Technology, Jonnada, Vzm
}

\begin{abstract}
We present a new image smoothing method by eliminating a manageable degree of low-amplitude structures while sharpening major edges by increasing the steepness of transition of edges. The use of low gradient minimization globally controls how many non-zero gradients are resulted in to approximate prominent structure in a sparsity control manner. Our method doesn't depend on local features like other edge-preserving methods.
\end{abstract}

Keywords: Filtering, gradient minimization, L0 smoothing,, sparsity.

\section{Introduction}

Images comprise well-structured and rich visual information. Edges are effective and expressive stimulation for neural interpretation of human visualization to make best sense of scene. Research follows on manipulating and understanding pictures, high-level inference with regard to salient structures and usefulness in a wide range of applications, including image recognition, segmentation, object classification, and many other photo editing and non-photorealistic rendering tasks.

A new editing method is presented which greatly helpful for enhancing image salient edges while diminishing insignificant details. It enables faithful principal structure representation by globally maintaining and possibly enhancing the most prominent set of edges by increasing steepness of transition by preserving the overall acutance.

Edge-preserving smoothing algorithms ${ }^{[1],[2],[3],[4],[5]}$ that aim to retain primary color change but our algorithm differs from these in essence in focuses on sparse gradient Counting scheme. The main focus of algorithm is to confine the discrete number of intensity changes among neighbouring pixels, which links mathematically to the $L 0$ norm for information sparsity pursuit. This idea also leads to an unconventional global optimization procedure involving a discrete metric, whose solution enables diversified edge manipulation according to saliency. Our method easily detects thin salient edges and more visually distinct.

\section{Background Work}

Local filtering algorithms like bilateral filters ${ }^{[1]}$ and its accelerated versions ${ }^{[6],[7],[8]}$ do edge-preserving smoothing. Edge preserving algorithms are also presented based on weighted least square optimization ${ }^{[9]}$ and envelope extraction ${ }^{[10]}$.

Due to simplicity and effectiveness of Bilateral filtering, it is widely used in removing noise-like structures. Trade off between flattening details and sharp edge preservation is provided by this method ${ }^{[9]}$. Anisotropic diffusion ${ }^{11}$ focuses on suppressing noise while preserving important structures, which involves an edge-stopping function to prevent smoothing from crossing strong edges in the image. The change of structures put together in a specific place and the output would converge to a constant-value image unless being stopped halfway.

Weighted least square (WLS) ${ }^{[9]}$, edge preserving regularization is more flexible with local filtering. Another edge preserving method, Total variation (TV) ${ }^{[12]}$ widely used to suppress noise. It influence contrast during smoothing process due to large gradient amplitude penalty. The best ways for image preservation by local signal extremes and used edge-aware interpolation to compute envelopes ${ }^{[10]}$. By using 1D Hilbert-Huang transform (HHT).a smoothed mean layer is extracted by averaging the envelopes. It aims to remove small scale oscillations.

Our idea targets globally preserving salient structures, even if they are small in resolution of concentration. One of the histogram smoothing process, to accelerate local filtering and proposed the mode-based filters ${ }^{[5]}$. Most recently, one of the image preserving method was demonstrated that multi-scale detail manipulation can be achieved using a modified Laplacian pyramid with coefficient classification ${ }^{13}$. Our method quite differs from above methods in overall estimation process. We emphasis our method as complementary to prior smoothing approaches.

Finally, interactive image editing methods, Graph-cut based methods ${ }^{[14],}{ }^{[15],}{ }^{[16]}$ and segmentation methods ${ }^{[17]}$, ${ }^{[18]}$ needs to select regions of interest in image with accurate boundaries levels. User interactions can be performed more efficient manner on our edge-enhanced images after removing low-amplitude structures. 
$L 0$ norm in sparse coding was proposed ${ }^{[20]}$ to extract sparse descriptors from natural images. Continuous $L_{p}$ norm with $p=1$ was enforced in total variation (TV) smoothing to suppress noise which penalize the magnitudes of salient edges. $L_{p}$ norm regularization with $0.5 \leq p \leq 1$ was also employed ${ }^{[21]}$ to model the sparsity of natural image gradients. A selective penalizing image gradient ${ }^{[22]}$ is related to the Weak Membrane model, which explicitly represents discontinuity and adjusts gradients only in continuous regions.

\subsection{Smoothing}

\section{Smoothing}

Enhancement of highest-contrast edges is done by confining the number of nonzero gradients, and smoothing is achieved in a global manner.

We denote the input discrete signal by $g$ and its smoothed result by $f$. Our method counts changes in amplitude discretely, written as:

$$
c(f)=\left\{p\left|f_{p}-f_{p+1}\right| \neq 0\right\}---(1)
$$

Where $p$ and $p+1$ are index neighbouring pixels. Gradient with respect to $\mathrm{p}$ in forward difference is $\left|f_{p}-f_{p+1}\right|$. The counting operator $\#\{\}$ counts the no of pixels that satisfies $\left|f_{p}-f_{p+1}\right| \neq 0$. that is, the $L 0$ norm of gradient. $c(f)$ counts the no of pixels not the gradient magnitude.

Our method uses this with constraint stating that the result $\mathrm{f}$ should be structurally similar to the input signal $g$. We express the specific objective function as:

$$
\min _{f} \sum_{p}\left(f_{p}-g_{p}\right)^{2} \text { s.t.c }(f)=k---(2)
$$

$k$ non-zero gradients exists in the result if $\mathrm{c}(\mathrm{f})=\mathrm{k}$. Structural information can be abstracted from eq(2). A larger $k$ yields a finer approximation, still characterizing the most prominent contrast.

In Equation (2), the quadratic intensity difference term $\left(f_{p}-g_{p}\right)^{2}$, That many pixels drastically change their colour. So low-amplitude structures can be primarily removed. Salient edges are automatically prevented from diminishing. The feature of this work is that no edge blurriness will be caused if filtering is avoided also with any value of $\mathrm{k}$.

For 2D images with different resolutions $\mathrm{k}$ value in Equation (2) may range from tens to thousands, especially in 2D images with different resolutions. To balance between structure flattening and result with same input, a general form is employed, and writes it as:

$$
\min _{f} \sum_{p}\left(f_{p}-g_{p}\right)^{2}+\lambda \cdot c(f)---(3)
$$

Where $\lambda$ is a weight directly controlling the significance of $c(f)$, which is nothing but smoothing parameter. Larger $\lambda$ value results in fewer edges in result. The number of non-zero gradients is monotone with respect to $1 / \lambda$.

\section{2 D Smoothing \& Formulation}

In 2D image representation, I denotes the input image and $S$ denotes the computed result. The color difference between neighbouring pixels along the $x$ and $y$ directions is calculated for each pixel as gradient $\nabla S_{p}=$ $\left(\partial_{x} S_{p}, \partial_{y} S_{p}\right)^{T}$.

Gradient measure is expressed as:

$$
\mathrm{C}(\mathrm{S})=\#\left\{\mathrm{p}\left|\partial_{\mathrm{x}} \mathrm{S}_{\mathrm{p}}\right|+\left|\partial_{\mathrm{y}} \mathrm{S}_{\mathrm{p}}\right| \neq 0\right\}---(4)
$$

$\mathrm{C}(\mathrm{S})$ counts $p$ whose magnitude $\left|\partial_{x} S_{p}\right|+\left|\partial_{y} S_{p}\right|$ is not zero.

$S$ can be estimated by solving

$$
\min _{S}\left\{\sum_{p}\left(S_{p}-I_{p}\right)^{2}+\lambda . C(S)\right\}---(5)
$$

For color images, the gradient magnitude $\left|\partial S_{p}\right|$ is defined as the sum of gradient magnitudes in red, green, blue. Equation (5) solution is difficult because the two terms model respectively the pixel-wise difference and global discontinuity statistically. An optimization strategy with half quadratic splitting is adopted to solve Equation (5). The idea of introducing auxiliary variables to expand the original terms and update them iteratively is used in half quadratic splitting. Splitting scheme ${ }^{19}$ used to solve a different convex problem. Due to the discrete nature of Equation (5), solution contains new sub problems. The solution is an approximation, which makes the problem easier to tackle and upholding the property to maintain and enhance salient structures.

An auxiliary variables $h_{p}$ and $v_{p}$, are introduced corresponding to $\partial_{x} S_{p}$ and $\partial_{y} S_{p}$ respectively, and rewrite the objective function as: 


$$
\min _{S, h, v}\left\{\sum_{p}\left(S_{p}-I_{p}\right)^{2}+\lambda C(h, v)+\beta\left(\left(\partial_{x} S_{p}-h_{p}\right)^{2}+\left(\partial_{y} S_{p}-V_{p}\right)^{2}\right)\right\}--(6)
$$

Where $C(h, v)=\#\left\{p\left|h_{p}\right|+\left|v_{p}\right| \neq 0\right\}$ and $\beta$ is an automatically adapting parameter to control the similarity between variables $(h, v)$ and their corresponding gradients.

Equation (6) approaches Equation (5) when $\beta$ is large enough. Equation (6) is solved through alternatively minimizing $(h, v)$ and $S$. In each pass, one set of the variables are fixed with values obtained from the previous iteration.

\subsubsection{Computing $S$}

Minimizing Equation (7) by omitting the terms not involving $\mathrm{S}$ in Equation (6) gives $\mathrm{S}$ estimation:

$$
\left\{\sum_{p}\left(\mathrm{~S}_{\mathrm{p}}-\mathrm{I}_{\mathrm{p}}\right)^{2}+\beta\left(\left(\partial_{\mathrm{x}} \mathrm{S}_{\mathrm{p}}-\mathrm{h}_{\mathrm{p}}\right)^{2}+\left(\partial_{\mathrm{y}} \mathrm{S}_{\mathrm{p}}-\mathrm{v}_{\mathrm{p}}\right)^{2}\right)\right\}--(7)
$$

The function is quadratic and thus has a global minimum even by gradient decent.

Derivative operators after Fast Fourier Transform (FFT), yields solution:

$$
S=\mathfrak{I}^{-1}\left(\frac{\mathfrak{I}(I)+\beta\left(\mathfrak{I}\left(\partial_{x}\right)^{*} \mathfrak{I}(h)+\mathfrak{I}\left(\partial_{y}\right)^{*} \mathfrak{I}(v)\right)}{\mathfrak{I}(1)+\beta\left(\mathfrak{I}\left(\partial_{x}\right)^{*} \mathfrak{I}\left(\partial_{x}\right)+\mathfrak{I}\left(\partial_{y}\right)^{*} \mathfrak{I}\left(\partial_{y}\right)\right)}\right)--(8)
$$

Where $\mathfrak{I}$ is the FFT operator and $\mathfrak{I}()^{*}$ denotes the complex conjugate. $\mathfrak{I}(1)$ is the Fourier Transform of the delta function. Computation in the Fourier domain is much faster than direct minimization of Equation (7) in the image space, which involves very-large-matrix inversion.

3.2.2 Computing (h,v)

The objective function for $(h, v)$ is

$$
\min _{h, v}\left\{\sum_{p}\left(\left(\partial_{x} S_{p}-h_{p}\right)^{2}+\left(\partial_{y} S_{p}-v_{p}\right)^{2}\right)+\frac{\lambda}{\beta} C(h, v)\right\}--(9)
$$

Where $C(h, v)$ returns the number of non-zero elements in $|h|+|v|$. The solution is easy because Equation (9) can be spatially decomposed where each element $h_{p}$ and $v_{p}$ can be estimated individually.

Eq. (9) is accordingly decomposed to

$$
\left.\sum_{p} \min _{h p, p p}\left\{\left(h_{p}-\partial_{x} S_{p}\right)^{2}+\left(v_{p}-\partial_{y} S_{p}\right)^{2}+\frac{\lambda}{\beta} H\left(\left|h_{p}\right|+\mid v_{p}\right)\right\}\right\}--(
$$

Where $H\left(\left|h_{p}\right|+\left|v_{p}\right|\right)$ is a binary function returning 1 if $\left|h_{p}\right|+\left|v_{p}\right| \neq 0$ and 0 otherwise. Each single term with respect to pixel $p$ in Eq. (10) is

$$
E_{p}=\left\{\left(h_{p}-\partial_{x} S_{p}\right)^{2}+\left(v_{p}-\partial_{y} S_{p}\right)^{2}+\frac{\lambda}{\beta} H\left(h_{p}|+| v_{p} \mid\right)\right\}--(11)
$$

Which reaches its minimum $E_{p}^{*}$ under the condition

\subsubsection{Proof}

$$
\left(h_{p}, v_{p}\right)=\left\{\begin{array}{l}
(0,0) i f\left(\partial_{x} S_{p}\right)^{2}+\left(\partial_{y} S_{p}\right)^{2} \leq \frac{\lambda}{\beta}--(12) \\
\left(\partial_{x} S_{p}, \partial_{y} S_{p}\right) \text { otherwise }
\end{array}\right.
$$

1) When $\frac{\lambda}{\beta} \geq\left(\partial_{x} S_{p}\right)^{2}+\left(\partial_{y} S_{p}\right)^{2}$, non-zero $\left(h_{p}, v_{p}\right)$ yields:

$$
E_{p}\left(\left(h_{p}, v_{p}\right) \neq(0,0)\right)=\left\{\begin{array}{l}
\left(h_{p}-\partial_{x} S_{p}\right)^{2}+\left(v_{p}-\partial_{y} S_{p}\right)^{2}+\frac{\lambda}{\beta} \\
\geq \frac{\lambda}{\beta} \\
\geq\left(\partial_{x} S_{p}\right)^{2}+\left(\partial_{y} S_{p}\right)^{2}
\end{array}\right.
$$

Note that $\left(h_{p}, v_{p}\right)=(0,0)$ leads to

$E_{p}\left(\left(h_{p}, v_{p}\right)=(0,0)\right)=\left(\partial_{x} S_{p}\right)^{2}+\left(\partial_{y} S_{p}\right)^{2}--(14)$ 
The minimum energy is produced when $\left(h_{p}, v_{p}\right)=(0,0)$.

Minimum energy is given by:

$$
E_{p}^{*}=\left(\partial_{x} S_{p}\right)^{2}+\left(\partial_{y} S_{p}\right)^{2}
$$

2) When $\left(\partial_{x} S_{p}\right)^{2}+\left(\partial_{y} S_{p}\right)^{2}>\lambda / \beta$ and $\left(h_{p}, v_{p}\right)=(0,0)$, Eq. (14) still holds. But $E_{p}\left(\left(h_{p}, v_{p}\right) \neq(0,0)\right)$ has its minimum value $\lambda / \beta$ when $\left(h_{p}, v_{p}\right)=\left(\partial_{x} S_{p}, \partial_{y} S_{p}\right)$. Comparing these two values, the minimum energy $E_{p}^{*}=\lambda / \beta$ is produced when $\left(h_{p}, v_{p}\right)=\left(\partial_{x} S_{p}, \partial_{y} S_{p}\right)$.

Compute the minimum energy $E_{p}{ }^{*}$ for each pixel $\mathrm{p}$ and Summing all of them, i.e., $\Sigma_{p} E_{p}{ }^{*}$, yields the global optimum for Equation (10).

Algorithm

Input: Image $\mathrm{I}$, Smoothing weight $\lambda$, parameters $\beta_{0}, \beta_{\max }$ and rate $\mathrm{k}$.

Initialization: $\mathrm{S} \leftarrow \mathrm{I}, \beta \leftarrow \beta_{0}, \mathrm{i} \leftarrow 0$

Repeat

With S(i), solve for hp(i) and vp(i) in eq (12).

With $\mathrm{h}(\mathrm{i})$ and $\mathrm{v}(\mathrm{i})$, solve for $\mathrm{S}(\mathrm{i}+1)$ with eq(8).

$$
\beta \leftarrow \mathrm{k} \beta, \mathrm{i}++ \text {. }
$$

Until $\beta \geq \beta_{\max }$

Output: result image S.
Parameter $\boldsymbol{\beta}$ starts from a small value $\beta_{0}$, it is multiplied by $\kappa$ each time in iterations. $\beta_{0}$ and $\beta_{\max }$ have fixed values $2 \lambda$ and $1 E 5$ respectively. $\kappa$ that is set to 2 is a good balance between efficiency and performance. When $\kappa$ set to 1.05 , produces higher-quality results. The parameter $\lambda$ is adjusted to control the level of structure coarseness.

\section{Experiment Results}

We compare our smoothing method with Bilateral Filter to evaluate the performance.

We use $2 \mathrm{D}$ example ${ }^{3}$ as input image as shown in Fig1. By varying the $\lambda$ value smoothing performance can be achieved more efficiently, the results are shown in Fig2 to Fig6. Fig7 shows the result of Bilateral Filter.

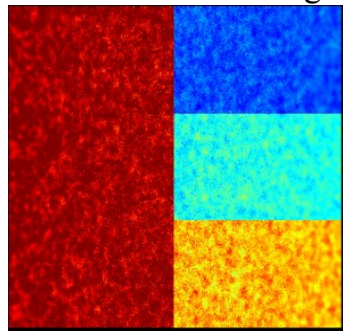

Fig1. Input image

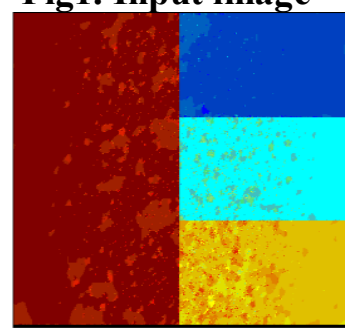

Fig3. With $\lambda=0.05$

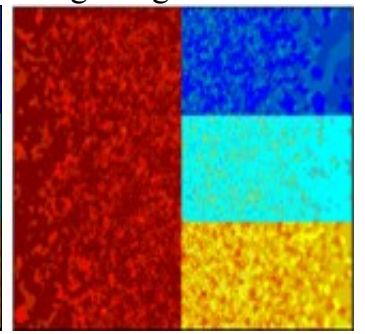

Fig2. With $\lambda=0.01$

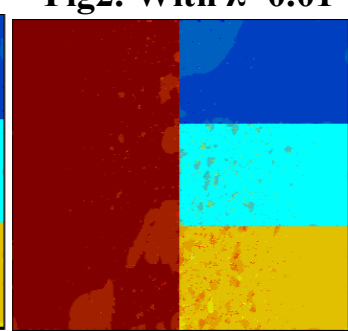

Fig4. With $\lambda=0.1$

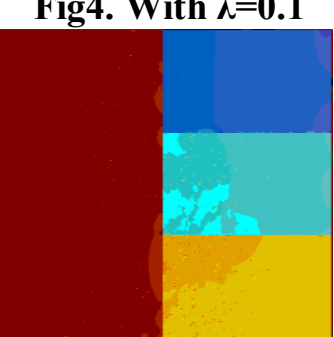

Fig5. With $\lambda=0.2 \quad$ Fig6. With $\lambda=2$ 


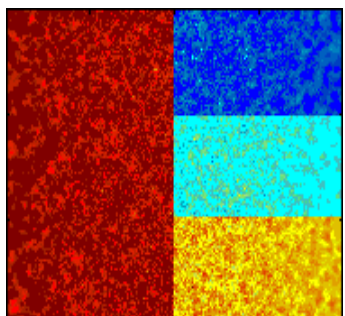

Fig7. Result of bilateral filter

Fig8 shows the input image. Fig9 shows the result of our method and Fig10 shows the result of Bilateral Filter.

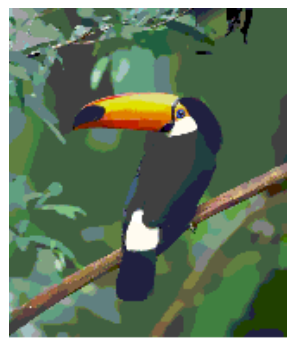

Fig8. Input image

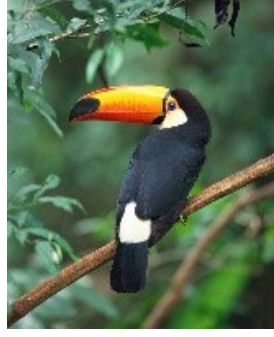

Fig9.with $\lambda=0.015$

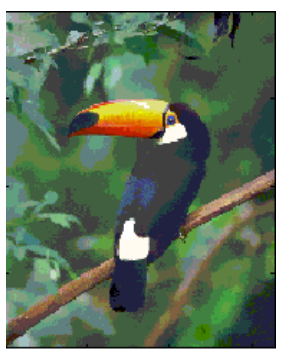

Fig10. Bilateral filter result

\section{Conclusion}

The proposed method smoothes the natural images based on removal of low-amplitude structures and preserval of salient edges. When our method combined with local filtering it produces very good results. Bilateral filtering blurs main boundaries under strong smoothing. First apply bilateral filtering followed by our method in order to achieve globally sharpen prominent edges.

\section{References}

[1] Tomasi,C.,And Manduchi, R. 1998. Bilateral Filtering For Gray And Color Images. In ICCV, 839-846.

[2] Durand, F.And Dorsey, J. 2002. Fast Bilateral Filtering For The Display Of High-Dynamic-Range Images. ACM Trans. Graph. 21, 3, 257-266.

[3] Farbman,Z.,Fattal,R.,Lischinski,D.,And Szeliski,R.2008. Edge-Preserving Decompositions For Multi-Scale Tone And Detail Manipulation. ACM Trans. Graph. 27, 3.

[4] Subr, K., Soler, C., And Durand, F. 2009. Edge-Preserving Multiscale Image Decomposition Based On Local Extrema. ACM Trans. Graph. 28, 5.

[5] Kass, M., and Solomon, J. 2010. Smoothed Local Histogram Filters. ACM Trans. Graph. $29,4$.

[6] Paris, S., And Durand, F. 2006. A Fast Approximation Of The Bilateral Filter Using A Signal Processing Approach. In ECCV (4), $568-580$.

[7] Weiss, B. 2006. Fast Median And Bilateral Filtering. ACM Trans. Graph. 25, 3, 519-526.

[8] Chen, J., Paris, S., And Durand, F. 2007. Real-Time Edge Aware Image Processing With The Bilateral Grid. ACM Trans. Graph. 26, 3, 103 .

[9] Farbman, Z., Fattal, R., Lischinski, D., And Szeliski, R. 2008. Edge-Preserving Decompositions For Multi-Scale Tone And Detail Manipulation. ACM Trans. Graph. 27, 3.

[10] Subr, K., Soler, C., And Durand, F. 2009. Edge-Preserving Multiscale Image Decomposition Based On Local Extrema. ACM Trans. Graph. 28, 5.

[11 ] Perona, P., And Malik, J. 1990. Scale-Space And Edge Detection Using Anisotropic Diffusion. IEEE Trans. Pattern Anal. Mach. Intell. 12, 7, 629-639.

[12] Rudin, L., Osher, S., And Fatemi, E. 1992. Nonlinear Totalvariation Based Noise Removal Algorithms. Physica D: Nonlinear Phenomena 60, 1-4, 259-268

[13] Paris, S., Hasinoff, S.W., And Kautz, J. 2011. Local Laplacian Filters: Edge-Aware Image Processing With A Laplacian Pyramid. ACM Trans. Graph.

[14] Rother, C., Kolmogorov, V., And Blake, A. 2004. "Grabcut": Interactive Foreground Extraction Using Iterated Graph Cuts. ACM Trans. Graph. 23, 3, 309-314. 
[15] Li, Y., Sun, J., Tang, C.-K., And Shum, H.-Y. 2004. Lazy Snapping. Acm Trans. Graph. 23, 3, 303-308.

[16] Liu, J., Sun, J., And Shum, H.-Y. 2009. Paint Selection. Acm Trans. Graph. 28, 3.

[17] Maji, S., Vishnoi, N., And Malik, J. 2011. Biased Normalized Cuts. In Cvpr.

[18] Arbelaez, P., Maire, M., Fowlkes, C., And Malik, J. 2011. Contour Detection And Hierarchical Image Segmentation. Ieee Trans. Pattern Anal. Mach. Intell. 33, 898-916.

[19] Wang, Y., Yang, J., Yin, W., And Zhang, Y. 2008. A New Alternating Minimization Algorithm For Total Variation Image Reconstruction. Siam J. Imaging Sciences 1, 3, 248-272.

[20] Mairal, J., Bach, F., Ponce, J., Sapiro, G., And Zisserman, A. 2009. Non-Local Sparse Models For Image Restoration. In ICCV, $2272-2279$.

[21] Levin, A., Fergus, R., Durand, F., And Freeman, W. T. 2007. Image And Depth From A Conventional Camera With A Coded Aperture. ACM Trans. Graph. 26, 3, 70.

[22] Blake, A., And Zisserman, A. 1987. Visual Reconstruction. The Mit Press. 